\title{
INTEGRAL OPERATORS OF CERTAIN UNIVALENT FUNCTIONS
}

\author{
O. P. AHUJA \\ Department of Mathematics \\ University of Papua New Guinea \\ Box 320, University P.O. \\ Papua New Guinea \\ (Received October 1, 1984)
}

ABSTRAT. A function $f$, analytic in the unit disc $\Delta$, is said to be in the family $R_{n}(\alpha)$ if $\operatorname{Re}\left\{\left(z^{n} f(z)\right)^{(n+1)} /\left(z^{n-1} f(z)\right)^{(n)}\right\}>(n+\alpha) /(n+1)$ for some $\alpha(0=\alpha<1)$ and for all $z$ in $\Delta$, where $n \in$ No, No $=\{0,1,2, \ldots\}$. The The class $R_{n}(\alpha)$ contains the starlike functions of order $\alpha$ for $n \geq 0$, and the convex functions of order $\alpha$ for $n \geq 1$. We study a class of integral operators defined on $R_{n}(\alpha)$. Finally an argument theorem is proved.

KEY WORDS AND PHRASES: Univalent, convolution, starlike, convex 1980 AMS SUBJECT CLASSIFICATION CODES: Primary 30C45, 30C99; Secondary 30055.

I INTRODUCTION.

Let A denote the family of functions $f$ which are analytic in the unit disc $\Delta=\{z:|z|<1\}$ and normalised such that $f(0)=0=f^{\prime}(0)-1$. The Hadamard product or convolution of two functions $f, g \in A$ is denoted by $f * g$. Let $D^{n} f=\left(z /(1-z)^{n+1}\right) * f, n \in$ No $=\{0,1,2, \ldots\}$ which implies that

$$
D^{n} f=z\left(z^{n-1} f\right)(n) / n !, n \in \text { No } .
$$

Denote by $S *(\alpha)$ and $K(\alpha)$ the subfamilies of $A$ whose members are, respectively, starlike of order $\alpha$ and convex of order $\alpha, 0 \leq \alpha<1$. Then

$$
\begin{aligned}
& \text { f } \varepsilon \mathrm{S} *(\alpha) \Leftrightarrow \operatorname{Re}\left(D^{1} \mathrm{f} / \mathrm{D}^{\circ} \mathrm{f}\right)>\alpha, z \varepsilon \Delta, \\
& \text { f } \varepsilon \mathrm{K}(\alpha) \Leftrightarrow \operatorname{Re}\left(D^{2} \mathrm{f} / \mathrm{D}^{1} \mathrm{f}\right)>(1+\alpha) / 2, \mathrm{z} \varepsilon \Delta
\end{aligned}
$$

Ruscheweyh [16] introduced the classes $\left\{K_{n}\right\}$ of functions $f \in A$ which satisfy the condition

$$
\operatorname{Re}\left(D^{n+1} f / D^{n} f\right)>\frac{1}{2}, z \varepsilon \Delta
$$

so that the definition of $K_{n}$ is a natural extension of $S *(1 / 2)$, and $K(0)$ He proved that $\mathrm{K}_{\mathrm{n}+1} \subset \mathrm{K}_{\mathrm{n}}$ for each $\mathrm{n} \varepsilon \mathrm{N}_{\mathrm{O}}$ Since $\mathrm{K}_{\mathrm{O}}=\mathrm{S} *(1 / 2)$, the elements of $K_{n}$ are univalent and starlike of order $1 / 2$. 
satisfy the condition

$$
\operatorname{Re}\left(z\left(D^{n} f\right)^{\prime} / D^{n} f\right)>\alpha, z \in \Delta
$$

for some $\alpha(0 \leq \alpha<1)$ We denote these classes by $R_{n}(\alpha)$ We have $\mathrm{R}_{\mathrm{o}}(\alpha)=\mathrm{S} *(\alpha)$ and $\mathrm{R}_{1}(\alpha)=\mathrm{K}(\alpha)$ for $0 \leq \alpha<1$. The classes $\mathrm{R}_{\mathrm{n}}=\mathrm{R}_{\mathrm{n}}(0)$ were considered earlier by Singh and Singh [17]. It is readily seen that for each $n \geq 0, R_{n}(\alpha) \subset R_{n}(0)$ and for each $n \geq 1, R_{n}(\alpha) \subset k_{n}$ We note that in definition (1.2), restriction $\alpha \geq 0$ can be replaced by $\alpha \geq(1-n) / 2$ for each $n \geq 1$ and, further, that the negative choices of $\alpha$ permit us fully to partition $K_{n}$ into classes $R_{n}(\alpha) \subset K_{n}(n \geq 1)$ such that

$$
\begin{aligned}
& \cup R_{n}(\alpha)=K_{n} . \\
& \frac{1-n}{2} \leq \alpha<1
\end{aligned}
$$

It can be easily seen that $R_{n+1}(\alpha) \subset R_{n}(\alpha)$ for each $n \in N_{0}$ and for all $\alpha$. These inclusion relations establish that $R_{n}(\alpha) \subset S^{*}(\alpha)$ for each $\mathrm{n} \geq 0$ and $\mathrm{R}_{\mathrm{n}}(\alpha) \subset \mathrm{K}(\alpha)$ for each $\mathrm{n} \geq 1$.

An important problem in univalent functions is the following: Given a compact family $F$ and an operator $J$ defined on $F$, is $J(f) \varepsilon F$ for every $f \in F$ ? Libera [11] established that the operator

$$
J(f)=\frac{2}{z} \int_{0}^{z} f(t) d t
$$

preserves convexity, starlikeness, and close-to-convexity. Bernardi [5] greatly generalised Libera's results. Many authors $[1,2,7,8,12,15,17]$ studied operators of the form

$$
J(f)=\frac{1+\gamma}{z} \int_{0}^{z} t^{\gamma-1} f(t) d t,
$$

where $\gamma$ is a real (or complex) constant and $f$ belongs to some favoured class of univalent functions from A. Recently, operators (1 4) have been studied in more general form by Causey and White [6], Miller, Mocanu and Reade [14], Barnard and Kellogg [3], and Bajpai [2]

In this paper, we study a class of integral operators of the form (1.4) defined on our family $R_{n}(\alpha)$ We also obtain an argument theorem for the class $R_{n}(\alpha)$.

2. INTEGRAL OPERATORS.

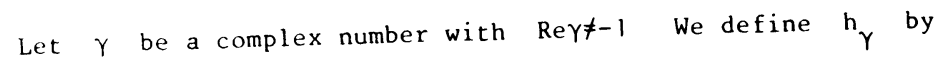




$$
h_{\gamma}(z)=\sum_{j=1}^{\infty} \frac{\gamma+1}{\gamma+j} z^{j}, z \in \Delta
$$

Let the operator $\mathrm{J}: \mathrm{A} \rightarrow \mathrm{A}$ be defined by $\mathrm{F}=\mathrm{J}(\mathrm{f})$, where

$$
F(z)=\frac{1+\gamma}{z} \int_{0}^{z} f(t) t^{\gamma-1} d t
$$

Then the function $F$ can also we written in the form

$$
F(z)=f(z) * h_{\gamma}(z)
$$

We need the following result of Jack [9] which is also due to Suffridge [18]

LEMMA. Let $w$ be nonconstant and analytic in $|z|<r<1, w(0)=0$ If $|w|$ attains its maximum value on the circle $|z|=r$ at $z_{0}$, then $z_{0} w^{\prime}\left(z_{0}\right)=k w\left(z_{0}\right)$, where $k$ is a real number and $k \geq 1$

We first give a condition on $f \in A$ for which the function $J(f)$ belongs to $R_{n}(\alpha)$

THEOREM 1. Let $0 \leq \alpha<1$, and $\gamma \neq-1$ be a complex constant such that $\operatorname{Re} \gamma \geq-\alpha, \operatorname{Im} \gamma \geq 0$, and $|\gamma|^{2}+2 \alpha(1+\operatorname{Re} \gamma) \geq 1$. If for a given $\mathrm{n} \varepsilon$ No, $\mathrm{f} \varepsilon \mathrm{A}$ satisfies the condition

$$
\operatorname{Re} \frac{z\left(D^{n} f(z)\right)^{\prime}}{D^{n} f(z)}>\alpha-\frac{(1-\alpha)(\alpha+\operatorname{Re} \gamma)}{2\left\{|\gamma|^{2}+2 \alpha \operatorname{Re} \gamma+\alpha^{2}+(1-\alpha) \operatorname{Im} \gamma\right\}}
$$

for all $z \in \Delta$, then $F(z)$ given by (2.2) belongs to $R_{n}(\alpha)$.

PROOF From (2.2), we obtain

$$
z\left(D^{n} F(z)\right)^{\prime}+\gamma D^{n} F(z)=(\gamma+1) D^{n} f(z)
$$

Define $w$ in $\Delta$ by

$$
\frac{z\left(D^{n} F(z)\right)^{\prime}}{D^{n} F(z)}=\frac{1+(2 \alpha-1) w(z)}{1+w(z)} \text {. }
$$

Here $w(z)$ is analytic in $\Delta$ with $w(0)=0$ and $w(z) \neq-1, z \varepsilon \Delta$ We need to show that $|w(z)|<1$ for all $z \varepsilon \Delta$. In view of $(2.4)$, (2.5) yields

$$
\frac{D^{n} f(z)}{D^{n} F(z)}=\frac{(1+\gamma)+(2 \alpha-1+\gamma) w(z)}{(1+\gamma)(1+w(z))}
$$




$$
\frac{z\left(D^{n} f(z)\right)^{\prime}}{D^{n} f(z)}=\alpha+(1-\alpha) \frac{1-w(z)}{1+w(z)}-\frac{2(1-\alpha) z w^{\prime}(z)}{(1+w(z))(1+\gamma+(2 \alpha-1+\gamma) w(z))}
$$

Now (2 7) should yield $|w(z)|<1$ for all $z \in \Delta$ for otherwise, there exists a point $z_{0} \in \Delta$ at which $\left|w\left(z_{0}\right)\right|=1$ and by Lemma, we have $z_{0} w^{\prime}(z)=k w\left(z_{0}\right), k \geq 1$. For this value of $z=z_{0}$, we find that (2.7) yields

$$
\begin{gathered}
\operatorname{Re}^{\frac{z_{Q}\left(D^{n} f\left(z_{O}\right)\right)^{\prime}}{D^{n} f\left(z_{O}\right)}}=\alpha-\frac{2 k(1-\alpha)(\alpha+\operatorname{Re} \gamma)}{\left|(1+\gamma)+(2 \alpha-1+\gamma) w\left(z_{0}\right)\right|^{2}} \\
\leq \quad \alpha-\frac{(1-\alpha)(\alpha+\operatorname{Re} \gamma)}{2\left\{|\gamma|^{2}+2 \alpha \operatorname{Re} \gamma+\alpha^{2}+(1-\alpha) \operatorname{Im} \gamma\right\}}
\end{gathered}
$$

which contradicts (2.3) Hence $|w(z)|<1$ for all $z \varepsilon \Delta$ and by (2.5), it follows that $F(z) \in R_{n}(\alpha)$.

COROLlaRY. If for a given $n \in N_{o}$, $f \in A$ satisties the condition

$$
\operatorname{Re} \frac{z\left(D^{n} f(z)\right)^{\prime}}{D^{n} f(z)}>\frac{2 \alpha(\gamma+\alpha)-(1-\alpha)}{2(\gamma+\alpha)}, z \in \Delta
$$

where $(\alpha, \gamma)$ is any point in the set

$$
D=\{(\alpha, \gamma): \gamma+2 \alpha \geq 1,0 \leq \alpha<1, \gamma>-1\},
$$

then $F(z)$ given by $(2.2)$ belongs to $R_{n}(\alpha)$.

PROOF. If $\gamma \neq-1$ is a real constant such that $\gamma+\alpha \geq 0$, then $|\gamma|^{2}+2 \alpha(1+\operatorname{Re} \gamma) \geq 1$ implies $(\gamma+1)(\gamma+2 \alpha-1) \geq 0$. The result follows from Theorem

It is easy to show that if $f \varepsilon R_{n}(\alpha)$, then $f$ satisfies the condition (2 3). Thus it follows from Theorem I that $J\left(R_{n}(\alpha)\right) \subset R_{n}(\alpha)$ More precisely, we state the result in

THEOREM 2 If $f \in R_{n}(\alpha)$, then the function

$$
J(f)=\frac{\gamma+1}{z^{\gamma}} \int_{0}^{z} f(t) t^{\gamma-1} d t
$$

is again an element of $R_{n}(\alpha)$, where $\gamma \neq-1$ is a complex constant with restrictions as stated in Theorem 1 .

REMARK 1 Letting $n=0=\gamma-1$ and $n=1=\gamma$, in Theorem 1 , we get $L(S *(\beta)) \subset S *(\alpha)$ and $L(K(\beta)) \subset K(\alpha)$ respectively, where $L$ is the Libera transform defined in (1.3), and

$$
B=\left(\left(2 \alpha^{2}+3 \alpha-1\right) / 2(1+\alpha)\right)<\alpha .
$$

These results improve the earlier results due to Libera [11] and Bernardi [5] in the sense that their results hold under much weaker conditions 
In [2], Bajpai has established that $J\left(S^{*}\right) \subset S^{*}(\alpha)$ for some $\alpha$. We generalize this result in

THEOREM 3. Let $\mathrm{J}: \mathrm{A} \rightarrow \mathrm{A}$ be defined as in (2 2), where $\gamma$ is a complex constant. If $f \in R_{n}$, then $J(f) \varepsilon R_{n}(\alpha)$, where $\alpha$ satisfies the inequality

$$
\alpha|| 1+\gamma|+| 2 \alpha-1+\gamma \mid]^{2} \leq 2(1-\alpha)(\alpha+\operatorname{Re} \gamma), \text { and } 0 \leq \alpha<1
$$

PROOF Proceeding as in Theorem 1 and applying Lemma, we have

$$
\begin{aligned}
\operatorname{Re} \frac{z_{0}\left(D^{n} f\left(z_{0}\right)^{\prime}\right.}{D^{n} f\left(z_{0}\right)} & \leq \alpha-\frac{2(1-\alpha)(\alpha+\operatorname{Re} \gamma)}{\left|(1+\gamma)+(2 \alpha-1+\gamma) w\left(z_{0}\right)\right|^{2}} \\
& \leq \alpha-\frac{2(1-\alpha)(\alpha+\operatorname{Re} \gamma)}{(|1+\gamma|+|2 \alpha-1+\gamma|)^{2}},
\end{aligned}
$$

where Rer $-\alpha$. Since the right hand side is $\leq 0$, we have a contradiction for $f \in R_{n} \equiv R_{n}(0)$. Thus we must have $|w(z)|<1$ for all $z$ in $\Delta$ and by (2 5), it follows that $J(f) \in R_{n}(\alpha)$.

REMARK I If we let $n=0=\gamma-1$ in the above theorem, then $L(S *) \subset S *\left(\frac{\sqrt{17}-3}{4}\right)$, where $L(f)=(2 / z) \int_{0}^{z} f(t) d t \quad$ Thus we have recovered a result of Miller, Mocanu and Reade ([14], pp 162-163).

REMARK 2 If $\mathrm{n}=1, \gamma$ is a real constant such that $\gamma+\alpha \geq 0$, and $\mathrm{f} \varepsilon \mathrm{K}$, then it follows from Theorem 3 that the function $F(z)$ in $(22)$ is an element of $\mathrm{K}(\alpha)$, where

$$
\alpha=\frac{-(2 \gamma+1)+\sqrt{(2 \gamma-1)^{2}+8(1+\gamma)}}{4} .
$$

This result was proved by Miller, Mocanu and Reade ([14], pp 165)

Further, this is an improvement of an earlier result due to Bernardi [5], who proved that $f \in K$ implies $F \in K$.

For $\gamma=n$, where $n \in N_{0}$, we have an improvement over Theorem 2

THEOREM 4. Let

$$
F(z)=f(z) * h_{n}(z)=\frac{n+1}{z^{n}} \int_{0}^{z} f(t) t^{n-1} d t
$$

If $f \in R_{n}(\alpha)$, then $F \in R_{n+1}(\alpha)$

PROC F. From (2.10), we obtain

$$
z\left(D^{n+1} F(z)\right)^{\prime}+n D^{n+1} F(z)=(n+1) D^{n+1} f(z)
$$




$$
z\left(D^{n} F(z)\right)^{\prime}+n D^{n} F(z)=(n+1) D^{n} f(z)
$$

Using the identity

$$
z\left(D^{n} f(z)\right)^{\prime}=(n+1) D^{n+1} f(z)-n D^{n} f(z)
$$

in (2.11) and (2.12), we obtain

$$
(n+1) D^{n+1} f(z)=(n+2) D^{n+2} F(z)-D^{n+1} F(z)
$$

and

$$
D^{n} f(z)=D^{n+1} F(z)
$$

In view of the identity $(213)$ and the relations $(2.14)$ and $(215)$, $f \in R_{n}(\alpha)$ yields

$$
\operatorname{Re}\left\{\frac{(n+2) D^{n+2} F(z)-(n+1) D^{n+1} F(z)}{D^{n+1} F(z)}\right\}>\alpha
$$

which implies that

$$
\operatorname{Re}\left\{\frac{z\left(D^{n+1} F(z)\right)^{\prime}}{D^{n+1} F(z)}\right\}>\alpha, \quad z \varepsilon \Delta
$$

This proves that $F \in R_{n+1}(\alpha)$.

REMARK For $\mathrm{n}=0$, Theorem 4 gives the well known result:

$J(S *(\alpha))=K(\alpha)$, where $J(f)=\int_{0}^{2}(f(t) / t) d t$

We now investigate the converse of Theorem 2. In fact, we find the sharp radius of the disc in which $f \in R_{n}(\beta)$ when $F$, defined in (2.2), is in $R_{n}(\alpha)$ for $0 \leq \alpha<1,0<\beta \leq 1$ : In [12], Libera and Livingston have solved this converse problem for the case $n=0, \gamma=1$ when $\alpha \leq \beta<1$. These authors were not able to obtain suitable results for the complementary case when $\beta<\alpha$ However, the method used in the next theorem gives results that are more general and also covers both $\beta \geq \alpha$ and $\beta<\alpha$.

THEOREM 5. If $F$ is an element of $R_{n}(\alpha)$ for $n \geq 0$ and $0 \leq \alpha<1$,

$$
F(z)=\frac{1+\gamma}{z^{\gamma}} \int_{0}^{z} f(t) t^{\gamma-1} d t
$$

with $z \in \Delta, \operatorname{Re} \gamma \geq-\alpha$, and $0 \leq \beta<1$, then the function $f$ is an element of $R_{n}(B)$ for $|z|<r_{0}$, where $r_{0}$ is the smallest positive root in $(0,1)$ of the equation

$$
(\gamma+2 \alpha-1)(2 \alpha-\beta-1) r^{2}+2((\gamma+\alpha)(\alpha-\beta)-(1-\alpha)(2-\alpha)) r+(\gamma+1)(1-\beta)=0
$$


PROOF Since $F \in R_{n}(\alpha)$, we can write

$$
\frac{z\left(D^{n} F(z)\right)^{\prime}}{D^{n} F(z)}=\alpha+(1-\alpha) P_{n}(z)
$$

where $P_{n}(z)$ is analytic in $\Delta$ and satisfies the conditions $P_{n}(0)=1$ $\operatorname{ReP}_{n}(z)>0$ for $z \in \Delta$ Using the identity

$$
z\left(D^{n} F(z)\right)^{\prime}=(n+1) D^{n+1} F(z)-n D^{n} F(z)
$$

in (2.18) and then taking logarithmic derivative, we obtain

$$
z\left(D^{n+1} F(z)\right)^{\prime}=D^{n+1} F(z)\left\{\alpha+(1-\alpha) P_{n}(z)+\frac{(1-\alpha) z P_{n}^{\prime}(z)}{n+\alpha+(1-\alpha) P_{n}(z)}\right\}
$$

From (2 16) we obtain

$$
z\left(D^{n+1} F(z)\right)^{\prime}+\gamma D^{n+1} F(z)=(\gamma+1) D^{n+1} f(z) .
$$

From (2 20) and (2 21) we have

$$
(\gamma+1) D^{n+1} f(z)=D^{n+1} F(z)\left[\alpha+\gamma+(1-\alpha) P_{n}(z)+\frac{(1-\alpha) z P_{n}^{\prime}(z)}{n+\alpha+(1-\alpha) P_{n}(z)}\right\}
$$

Also (2.18) together with the identity (2 4) yields

$$
(1+\gamma) D^{n} f(z)=D^{n} F(z)\left(\alpha+\gamma+(1-\alpha) P_{n}(z)\right) \text {. }
$$

Now from the relations (2 22), ( 2 23), and (2.18) we conclude that

$$
\frac{z\left(D^{n} f(z)\right)^{\prime}}{D^{n} f(z)}-\beta=\alpha-\beta+(1-\alpha) P_{n}(z)+\frac{(1-\alpha) z P_{n}^{\prime}(z)}{\alpha+\gamma+(1-\alpha) P_{n}(z)} \text {. }
$$

Using the well known estimates

$$
\left|\mathrm{zP}_{\mathrm{n}}^{\prime}(\mathrm{z})\right| \leq\left(2 \mathrm{r} /\left(1-\mathrm{r}^{2}\right)\right) \operatorname{ReP}_{\mathrm{n}}(\mathrm{z})
$$

and

$$
\operatorname{ReP}_{n}(z) \geq(1-r) /(1+r),|z|=r
$$

in (2 24), we obtain

$$
\operatorname{Re}\left[\frac{z\left(D^{n} f(z)\right)^{\prime}}{D^{n} f(z)}-\beta\right] \geq(\alpha-\beta)+\frac{(1-\alpha)((1-r)(\gamma+1+(\gamma+2 \alpha-1) r)-2 r)}{(1-r)((\gamma+2 \alpha-1) r+\gamma+1)}
$$

where $\operatorname{Re} \gamma \geq-\alpha$. Therefore,

$$
\operatorname{Re}\left\{\frac{z\left(D^{n} f(z)\right)^{\prime}}{D^{n} f(z)}\right\}>B
$$

if the right side of $(2.25)$ is positive, which is satisfied provided that $r<r_{0}$, where $r_{0}$ is the smallest positive root in $(0,1)$ of $(2.17)$.

The result in the theorem is sharp with the function $f$ defined by

$$
f(z)=(1 /(1+c)) z^{1-c}\left(z^{c} F(z)\right)^{\prime} \text {, }
$$


where

$$
\begin{aligned}
& c=\operatorname{Re} \gamma \geq-\alpha \text {, and } F \quad \text { is given by } \\
& z \frac{\left(D^{n} F(z)\right)^{\prime}}{D^{n} F(z)}=\frac{1-(2 \alpha-1) z}{1-z}
\end{aligned}
$$

REMARK. By specializing choices of $\alpha, \beta, \gamma$, and $n$, theorem 5 gives rise to the corresponding results obtained earlier in $[3,4,8,12,13,15]$ and by many others

3 AN ARGUMENT THEOREM.

THEOREM 6 If $f \in R_{n}(\alpha)$, then

$$
\left|\arg \frac{D^{k} f(z)}{z}\right| \leq 2(1-\alpha) \sin ^{-1} r+\sum_{m=0}^{k-1} \sin ^{-1}\left(\frac{2(1-\alpha) r}{m+1-(m+2 \alpha-1) r^{2}}\right)
$$

for each $k(0 \leq k \leq n+1)$.

PROOF We may write

$$
\frac{D^{k} f(z)}{z}=\frac{f(z)}{z} \prod_{m=0}^{k-1} \frac{D^{m+1} f(z)}{D^{m} f(z)}, 0 \leq k \leq n+1 \text {, }
$$

which yields

$$
\left|\arg \frac{D^{k} f(z)}{z}\right| \leq\left|\arg \frac{f(z)}{z}\right|+\sum_{m=0}^{k-1}\left|\arg \frac{D^{m+1} f(z)}{D^{m} f(z)}\right| .
$$

Since $R_{n+1}(\alpha) \subset R_{n}(\alpha) \forall n \in N_{0}$, it follows that $f \varepsilon R_{m}(\alpha)$ for each $\mathrm{m}(0 \leq \mathrm{m} \leq \mathrm{n}) \quad$ Setting

$$
\frac{D^{m+1} f(z)}{D^{m} f(z)}=q_{m}(z), \quad(0 \leq m \leq n)
$$

we note that $\operatorname{Re}\left(q_{m}(z)\right) \geq(m+\alpha) /(m+1)$

Therefore, the function

$$
\begin{aligned}
w(z) & =\frac{\left(q_{m}(z)-\frac{m+\alpha}{m+1}\right)-\left(1-\frac{m+\alpha}{m+1}\right)}{\left(q_{m}(z)-\frac{m+\alpha}{m+1}\right)+\left(1-\frac{m+\alpha}{m+1}\right)} \\
& =\frac{q_{m}(z)-1}{q_{m}(z)-\left(\frac{2(m+\alpha)}{m+1}-1\right)}
\end{aligned}
$$

is analytic with $w(0)=0$ and $|w(z)|<1$ in $\Delta$ Hence by Schwarz's Lemma , 


$$
\left|\frac{q_{m}(z)-1}{q_{m}(z)+1-2(m+\alpha) /(m+1)}\right|<|z|
$$

for $z$ in $\Delta$ Now it is easy to see that the values of $q_{m}(z)$ are contained in the circle of Appolonius whose centre is at the point $\left(m+1-(m+2 \alpha-1) r^{2}\right) /\left((1+m)\left(1-r^{2}\right)\right)$ and has radius $2(1-\alpha) r /\left((m+1)\left(1-r^{2}\right)\right)$

Thus $\max \left|\arg q_{m}(z)\right|$ is attained at the points where

$$
\arg q_{m}(z)= \pm \sin ^{-1}\left(\frac{2(1-\alpha) r}{m+1-(m+2 \alpha-1) r}\right)
$$

which gives

$$
\left|\arg \frac{D^{m+1} f(z)}{D^{m} f(z)}\right| \leq \sin ^{-1}\left(\frac{2(1-\alpha) r}{m+1-(m+2 \alpha-1) r}\right)
$$

for $0 \leq m \leq n$, and $z \in \Delta$

Next, note that $R_{n}(\alpha)$, $S *(\alpha), n \geq 0$, and $f \in S *(\alpha)$ if and only

if $F(z)=\int(f(z) / z) d z$ is in $K(\alpha)$ But for $F \in K(\alpha)$, we have

$$
\left|\arg F^{\prime}(z)\right| \leq 2(1-\alpha) \sin ^{-1} r \quad(|z|=r)
$$

Thus $f \in \mathrm{R}_{\mathrm{n}}(\alpha)$ implies

$$
\left|\arg \frac{f(z)}{z}\right| \leq 2(1-\alpha) \sin ^{-1} r
$$

Applying ( 3 ) and (3.4) to (3.1) we obtain the result.

For $n=0$, we obtain

COROLLARY If f $\varepsilon S \div(\alpha)$, then (3.4)

and

$$
\left|\arg f^{\prime}(z)\right| \leq 2(1-\alpha) \sin ^{-1} r+\sin ^{-1}\left(\frac{2(1-\alpha) r}{1-(2 \alpha-1) r^{2}}\right)
$$

REMARK The case $\mathrm{n}=0, \alpha=0$ way proved by Krzyz [10].

The author is grateful to the referee for his suggestions which greatly helped in presenting this paper in a compact form.

\section{REFERENCES}

1. AL-AMIRI, H.S.: Certain generalizations of pre-starlike functions, J. Australian Math. Soc (Serie A) 28(1979), 325-334

2. BAJPAI, S.K : Spirallike integral operators, Internat. J. Math. \& Math. Sci., Vol 2. (1981), 337-351. 
3 BARNARD, R W and KELLOGG C.: Applications of convolution operators to problems in univalent function theory, Mich. Math J. 27 (1980), no 1, 81-94

4. BERNARDI, S D.: The radius of univalence of certain analytic functions, Proc Amer Math. Soc 24 (1970), 312-318.

5 BERNARDI, S.D.: Convex and starlike univalent functions, Trans. Amer. Math. Soc. 135 (1969), 429-446

6 CAUSEY, W.M and WHITE W.L.: Starlikeness of certain functions with integral representations, J. Math. Anal. Appl. 64 (1978), 458-466.

7. GOODMAN, A.W : Univalent functions, Vol II, Mariner Publishing Company, Inc, 1983.

8. GUPTA, V P. and JAIN, P K.: On starlike functions, Rendiconti di Mat 9 (1976), 433-437.

9 JACK, I S.: Functions starlike and convex of order $\alpha$, J. London Math Soc., 3(1971), 469-474.

$10 \mathrm{KRZYZ}, \mathrm{J} . \mathrm{E}$ On the derivative of close-to-convex of order $\alpha$, J. London Math. Soc., 3 (1971), 469-474.

11. LIBERA, J.R.: Some classes of regular univalent functions, Proc. Amer Math. Soc. 16 (1965), 755-758.

12 LIBERA, R.J. and LIVINGSTON, A.E.: On the univalence of some classes of regular functions, Proc. Amer. Math Soc. 30 (1971), 327-336

13 LIVINGSTON, A.E.: On the radius of univalence of certain analytic functions, Proc. Amer. Math. Soc. 17 (1966), 352-357.

14 MILLER, S.S., MOCANU, P.T. and READE, MAXWELL 0.: Starlike integral operators, Pacific J. Math. 79 (1978), 157-168

15. PADMANABHAN, K.S.: On the radius of univalence of certain classes of analytic functions, J. London Math. Soc. (2) 1 (1969), 225-231

16 RUSCHEWEYH, S.: New criteria for univalent functions, Proc. Amer. Math. Soc. $49(1975), 109-115$.

17. SINGH, R. and SINGH, S.: Integrals of certain univalent functions, Proc. Amer Math. Soc. 77 (1979), 336-340.

18 SUFFRIDGE, T.J.: Some remarks on convex maps of the unit disk, Duke Math J. 37 (1970), 775-777 


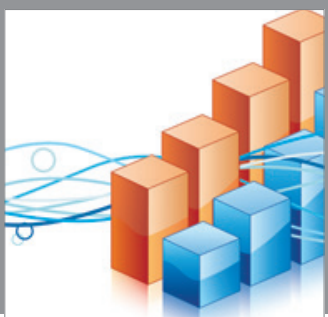

Advances in

Operations Research

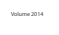

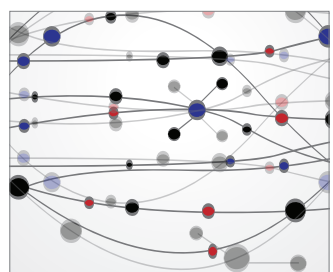

\section{The Scientific} World Journal
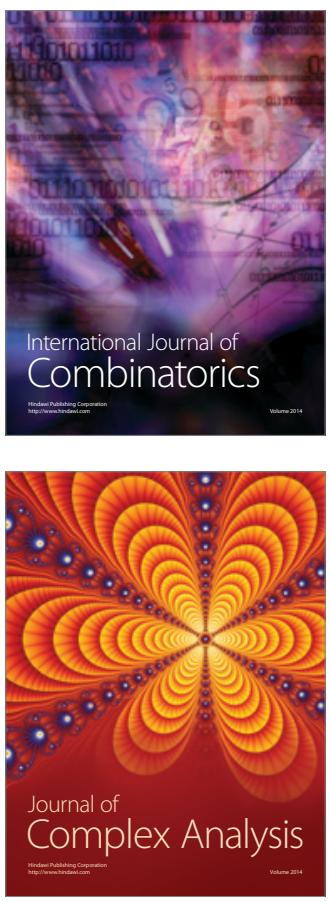

International Journal of

Mathematics and

Mathematical

Sciences
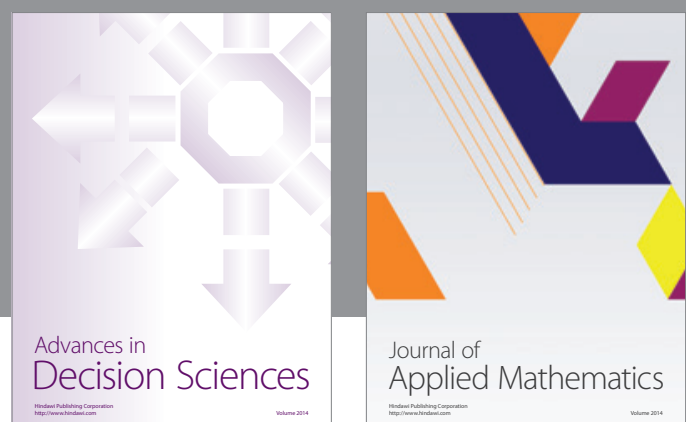

Journal of

Applied Mathematics
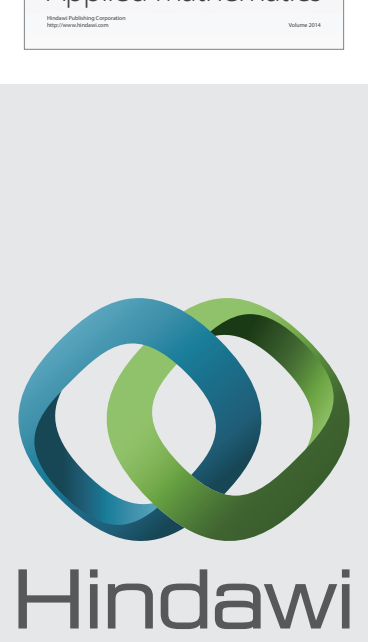

Submit your manuscripts at http://www.hindawi.com
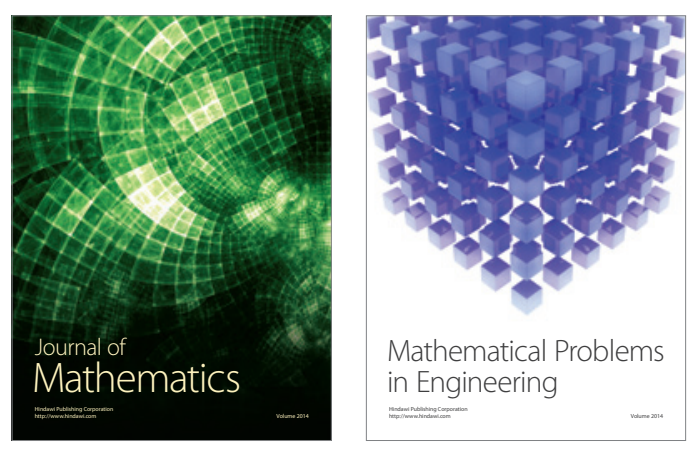

Mathematical Problems in Engineering
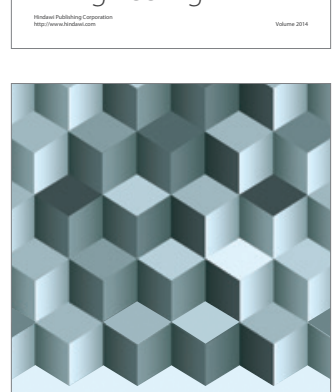

Journal of

Function Spaces
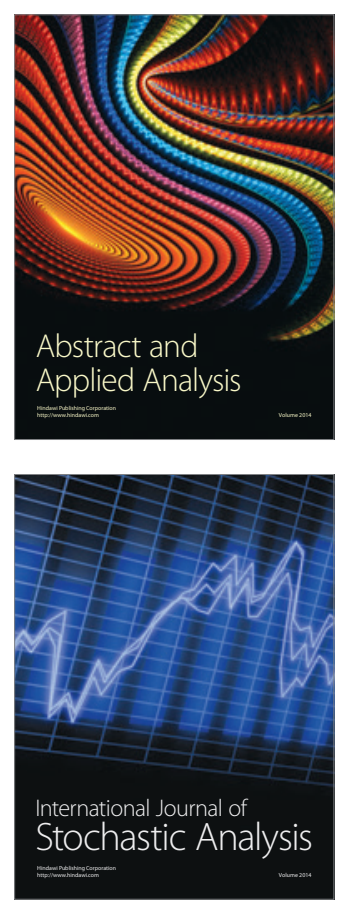

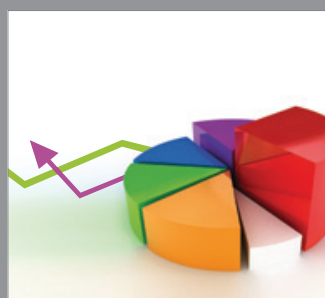

ournal of

Probability and Statistics

Promensencen
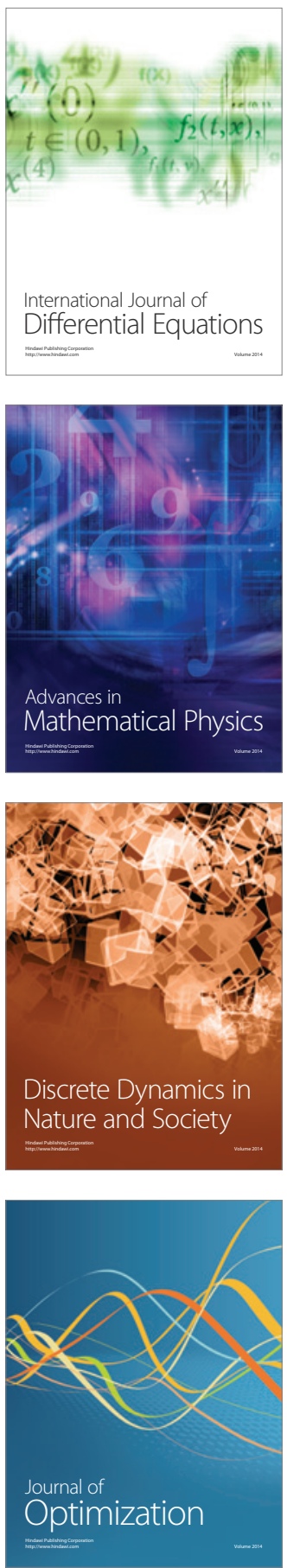